\title{
Brain tissue banks in psychiatric and neurological research
}

\author{
N J Cairns, P L Lantos
}

\section{Introduction}

Properly organised brain banks have a relatively recent history with only a few more than a decade old. ${ }^{1}$ Much recent progress in the understanding of the pathogenesis of both common and rare disorders of the nervous system has come about by the use of postmortem human brain tissue. This scientific progress has been based on the systematic collection and distribution by brain banks of brain tissue according to standardised clinical and pathological criteria. In order to be responsive to technological and scientific advances in medical research brain banks need to be located in centres with a strong academic and neuroscience base. Where material from rare familial cases or childhood disorders is scarce, cooperation with other brain banks may be necessary. Brain banks need to have policies for allocating rare and invaluable material as well as considering safety and ethical issues surrounding the use of donated human tissue for medical research. To be successful, brain banks need to liaise effectively and sensitively with a large constituency of donors, their families, nursing homes, general practitioners, hospital doctors, funeral directors, pathologists, mortuary staff, and, not least, the users of brain tissue. It is these doctors and scientists who are endeavouring to find the causes and more effective treatment for common neurodegenerative disorders, such as Alzheimer's disease and Parkinson's disease, where animal models are of uncertain value, and less common ones where there are no candidate genes implicated in the pathogenesis.

\section{Brain Bank,}

Department of

Neuropathology,

Institute of Psychiatry

Correspondence to: Professor P L Lantos, Brain Bank, Department of Neuropathology, Institute of Psychiatry, De Crespigny Park, London SE5 8AF.

Accepted for publication 4 June 1996 brain bank is to collect postmortem or biopsy material from clinically and pathologically wel characterised cases in a continuous and systematic manner to serve the needs of specific research programmes. For example, the London Brain Bank for Neurodegenerative Diseases, based in the Department of Neuropathology, Institute of Psychiatry, London, systematically collects brain tissue from dementia patients with Alzheimer's disease and related disorders and makes this tissue available to an Interdisciplinary Research Group on neurodegenerative disorders consisting of 60 psychiatrists, psychologists, neurologists, radiologists, neuropathologists, and basic laboratory scientists within the Institute of Psychiatry. In addition, research groups and individuals at other research centres, mainly in London, but also elsewhere in the UK and abroad, are also supplied with tissue from patients with neurodegenerative diseases and controls.

\section{Scientific achievements based on systematic brain banking}

Analyses of clinically and neuropathologically well characterised series of cryopreserved and formalin fixed human brain tissue have led to major discoveries in several psychiatric and neurological disorders. A decrease in the concentration of dopamine in specific brain regions in patients with Parkinson's disease led to the development of the therapeutic agent L-dopa. In Alzheimer's disease and dementia with Lewy bodies, the loss of cholinergic neurons and a reduction in an important brain enzyme, acetylcholinesterase, in postmortem brain tissue has led to cholinergic replacement therapy. ${ }^{2}$ A major component of the neurofibrillary tangle is a microtubule associated protein called tau. This protein has been extracted from Alzheimer's disease brains and found to be hyperphosphorylated. ${ }^{3}$ Molecular pathological studies have identified genes that are causative or risk factors, or both, in Alzheimer's disease ${ }^{4}$ Huntington's disease ${ }^{5}$ and motor neurone disease. ${ }^{6}$ Intact genomic DNA obtained from frozen or archival tissue can now be used to reassign unusual and atypical cases which may have abnormal genes in common, as well as rare disorders such as are found in prion disease. ${ }^{7}{ }^{8}$ The systematic collection of brains of patients who have died of AIDS has shown that neuronal loss is only associated 
with the symptomatic stage of the disease. ${ }^{9}$ These discoveries and the new techniques developed by brain banks have produced new insights into the understanding of the pathogenesis of psychiatric and neurological disorders as well as offering real hope for improved treatment.

\section{Clinical information}

The detailed clinical assessment of patients is an important prerequisite of brain banking and an essential component of any clinicopathological study. Users of tissue need confidence that the patient has been assessed and diagnosed in a reliable and systematic manner. A sound clinical diagnosis is particularly important with psychiatric patients, such as those with schizophrenia where the neuropathology, given the current state of knowledge, may be subtle. In a typical area based study, patients will be regularly assessed, ideally at least annually, until death. The clinician, in consultation with the brain bank, will seek permission from the patient or his or her family for a postmortem examination and removal of brain tissue and other organs for medical research. Ideally, standardised biochemical, medical, psychiatric, and neurological examinations will be carried out by experienced clinicians. Imaging (computed tomography, magnetic resonance imaging, functional magnetic resonance imaging, and positron emission tomography) may be performed if there are local facilities.

\section{Pathological protocols}

Brain tissue obtained either at biopsy or necropsy should be examined by a neuropathologist. This neuropathological assessment has two functions. Firstly, it is a means of validating the clinical diagnosis, arguably the most reliable audit of clinical diagnosis and treatment, and, secondly, it is a form of quality control of tissue provided to the neuroscience community. ${ }^{10}$ Making a neuropathological diagnosis is not always straightforward. Not uncommonly, the diagnosis, particularly with neurodegenerative disorders, depends on the estimation of the severity or density of a particular lesion, such as the numbers of neuritic plaques in Alzheimer's disease, and comparing this "pathological" density with "age related" changes. In addition, the diagnosis may be complicated by being dependent on the clinical diagnosis. For example, the diagnosis of Alzheimer's disease according to the criteria of Mirra $e t$ al $^{11}$ depends on a certain density of neuritic plaques within specified brain regions and the presence of clinical symptoms of dementia. In addition, more than one disease process may contribute to the dementing symptoms. For example, not infrequently in dementia with Lewy bodies, there are extensive Alzheimer type changes. In other cases, small infarcts may be present, sufficient for a differential diagnosis of cerebral vascular disease. The relative weight given to each lesion in explaining the clinical symptoms is further complicated by their location within the brain and by age related changes.
Consensus neuropathological criteria exist for an increasing number of psychiatric and neurological disorders including common disorders like Alzheimer's disease ${ }^{11}$ and rarer disorders, such as motor neurone disease, ${ }^{12}$ progressive supranuclear palsy, ${ }^{13}$ multiple system atrophy, ${ }^{14}$ and corticobasal degeneration. ${ }^{15}$ These criteria, as far as they apply to brain banking, specify minimal clinical criteria required, brain regions to be sampled, and histological stains necessary for a confident diagnosis. Immunohistochemistry plays an increasingly important role in the identification of hallmark lesions and other abnormalities in diseases of the central nervous system (CNS). The adoption of standardised neuropathological criteria permits intercentre comparisons and the collection and use of material for multicentre studies.

\section{Control cases}

Probably the most difficult task of any brain bank is finding suitable and sufficient numbers of control cases. It is widely recognised that it is far more difficult to obtain control material than pathological tissue. Brain tissue donors are more readily recruited under the auspices of disease related charities such as the Alzheimer's Disease Society and the Parkinson's Disease Society than are the neurologically and psychiatrically normal. Although the spouse of an affected donor may readily enter a donor programme, it may be many years before his or her wishes can be realised. In such cases, the psychiatric and neurological state can only be assessed by hospital or general practitioner records. Although the clinical data available may be of variable quality, the neuropathologist is at liberty to examine the brain thoroughly and to identify age related changes and exclude frank pathology. In this way, the brain tissue will be as reliably and consistently described as the pathology from a well characterised patient with dementia, for example.

\section{Tissue sampling and storage}

Systematic sampling of defined brain regions is mandatory for a neuropathological diagnosis according to established international protocols. The basic principle at this initial stage is to establish a reliable neuropathological diagnosis with the minimum number of tissue blocks and histological stains. However, where there is doubt over the clinical diagnosis or where additional pathology is revealed during the macroscopic examination of the brain, additional blocks of tissue may be taken to make a sound diagnosis. Clearly, the sampling of tissue will be influenced by the clinical diagnosis. While samples of the spinal cord will be necessary to confirm a diagnosis of motor neurone disease, the diagnosis of Alzheimer's disease can be made without these regions of the CNS. The requirements of tissue users will influence the manner in which the brain is dissected, processed, examined, and stored. Each brain bank has its own set of dissection protocols reflecting the disorders collected, the pathological protocols followed and research users' requirements. Typically, one half of the 
fresh brain is divided sagitally: one half is frozen for biochemistry and molecular genetic studies and the other half is fixed in formalin for histology. Various methods may be used to freeze the brain tissue (snap freezing in liquid nitrogen, cryoprotection and contact freezing with a brass plate at $-70^{\circ} \mathrm{C}$ ) depending on local needs and resources.

In addition to brain tissue, cerebrospinal fluid is often routinely taken postmortem and frozen for biochemical studies. In some disorders additional tissues may be taken such as peripheral nerves and muscles in motor neurone disease. In infections of the CNS, such as HIV, it may be informative to identify pathology and determine viral load and strain in multiple organs; in such cases a few grams of tissue from the organs of interest may be sufficient for biochemical and molecular genetic studies. Frozen tissues may be routinely stored for several years in $-70^{\circ} \mathrm{C}$ freezers. With invaluable material it is essential to have constant monitoring of the freezers and back-up facilities to cope with occasional power and mechanical failures.

\section{Databases}

A simple catalogue of material stored in a brain bank is not sufficient to carry out the wide ranging requests for tissue according to several variables. The relatively low cost of personal computers and appropriate software now permit the storage, manipulation and retrieval of large amounts of data. Typically, the software chosen (dBASE from Borland and ACCESS from Microsoft are popular packages) will be that which produces a relational database - that is, one in which all the data are interlinked across different variables. These databases can be modified to include data and text and tailored to local use by the nonspecialist. Both clinical signs and symptoms, cognitive and other test scores may be included as well as the type of tissue stored (frozen, formalin fixed, paraffin wax embedded), its brain region, histology, and neuropathological diagnosis. Commonly, clinical diagnoses are coded according to standardised criteria such as DSM-IV ${ }^{16}$ and ICD-10 ${ }^{17}$ and pathology according to the SNOMED classification. ${ }^{18}$ This coding may facilitate retrieval of tissues. In addition, there are several variables which the brain bank may need to control for when providing tissue to the research worker. These include: age, sex, brain weight, ${ }^{19}$ agonal state, ${ }^{2}$ and seasonal and circadian variation. In some studies, such as in schizophrenia, laterality may need to be taken into account. Postmortem factors that may affect tissue biochemistry include: postmortem delay, $\mathrm{pH}$ of brain tissue, methods of fixation and freezing, and the date and time stored. ${ }^{2021}$

\section{Tissue dissemination}

Requests for tissue must be made in writing from bona fide research workers in established research organisations including universities and industry. Unfixed frozen human brain tissue needs to be handled with care and users need to be informed of any special safety precautions that they should take. A reliable courier should be used where transport is over either short or long distances and a record of every brain tissue sample distributed must be made. Once a year users of tissue are asked to provide information on the use of tissue provided by the brain bank and copies of any publications making use of this tissue.

\section{Organisation}

It is important that the staff working for the brain bank are available at all hours of the day and night, during the working week and at weekends and bank holidays. Although postmortem examinations may be carried out during office hours, the majority of deaths occur outside these times, staff need to be available to speak to relatives, carers, nursing home staff, medical staff, and funeral directors in order to coordinate the brain tissue donation. In addition, staff need to be available at all times to deal with freezer failure. In practice, a rota operates in most brain banks where the designated individual on call carries a radio pager or mobile telephone.

The majority of brain banks are funded either directly or indirectly by central government and are seen as an essential service underpinning peer reviewed neuroscience research. In the UK, several brain tissue banks are funded by the Medical Research Council. A minority of brain banks are funded to a greater or lesser degree by non-profit making organisations such as the Parkinson's Disease Society and the Multiple Sclerosis Society.

For management purposes, the roles of the brain bank can be divided into a clinical role involving the prospective assessment of patients, usually by a consultant or senior clinician; and the pathological role, following death, involving the necropsy, dissection, tissue processing, diagnosis, storage, and distribution, which is overseen by a consultant or senior neuropathologist. As the director, the senior neuropathologist should manage the bank staff and decide on policy. The dissection of tissue may be performed by a qualified research worker, registrar or an experienced medical and laboratory scientific officer (MLSO). Liaison with patients, families, general practitioners, neuropathologists, mortuary staff, and others may be performed by an administrator who may be a research nurse or scientist. Technical work involved in processing and preparing histological sections is usually carried out by MLSOs. Family liaison, report writing and data entry, core banking tasks, are performed by an administrator or secretary.

\section{Safety}

A full risk assessment needs to be undertaken before the establishment of a brain bank and when new procedures are proposed. These procedures must follow established guidelines. ${ }^{22}{ }^{23}$ Staff should be immunised against hepatitis B and instructed in the safe handling of dangerous pathogens. Infective agents that may be present include HIV, hepatitis B and C, tuberculosis, syphilis, and prion protein from cases of Creutzfeldt-Jakob disease and related 
disorders. Infectivity is not destroyed by freezing and special care must be taken in dissecting and sectioning these hazardous tissues. ${ }^{22-28}$

\section{Ethical issues}

The retention of tissue at necropsy for research purposes other than to establish the cause of death is governed by the Human Tissue Act (1961). Consent for the removal of tissue for educational purposes or medical research is usually given by the donor or the next of kin, or when there are no relatives by the designated officer of the hospital where the patient has been cared for. The donation of human tissue after death should be unconditional and involve no inducement or payment. Brain banks act as stewards in the sense that they have responsibilities and obligations associated with ownership but without the rights associated with property. These duties include: the responsible use of data-for example, conforming to the Data Protection Act (1984), access to ethically approved research, and the responsible and respectful handling of data and tissue.

\section{Brain bank audit and review}

The funding of brain banks is regularly reviewed. The initial grant to establish a brain bank is made on the basis of peer review and is dependent on the quality of the neuroscience it underpins and reflects the strategic aims of the funding agency. Audit of the work of the brain bank includes the quality of clinical information, recruitment of cases, neuropathological assessment, distribution to users, and the contribution to basic and applied neuroscience research as demonstrated in publications.

\section{Brain banking in Europe in the decade of the brain}

Europe has an unrivalled reputation in brain research and it is no surprise that there are more brain banks in Europe than in any other continent. Significant advances in our understanding of psychiatric and neurological disorders has come about by the systematic collection of clinically and neuropathologically well characterised tissue. The coordination of brain banking activity in Europe is being promoted by a Concerted Action of the European Community. This network of brain banks has led to the establishment of common neuropathological criteria for the diagnosis of many psychiatric and neurological disorders. ${ }^{10}$ More recently, an American Brain Banking Network has been established which will, in time, accommodate the databases of the member banks. ${ }^{29}$ These European and American networks will enable more efficient collection of common disorders and make more efficient the coordination of the collection of tissue from rare disorders.
1 Cruz-Sanchez FF, Tolosa E. How to run a brain bank. $f$ Neural Transm 1993;39(Suppl).

2 Perry EK, Perry RH, Tomlinson BE. The influence of agonal state on some neurochemical activities of postmortem nal state on some neurochemical activities of pos

3 Hanger DP, Brion J-P, Gallo J-M, Cairns NJ, Luthert PJ, Anderton BH. Tau in Alzheimer's disease and Down's syndrome is insoluble and abnormally phosphorylated. Biochem $\mathcal{F}$ 1991;275:99-104.

4 Goate A, Chartier-Harlin M-C, Mullan M, Brown J, Crawford F, Fidani L, et al. Segregation of a missense mutation in the amyloid precursor protein gene with familial Alzheimer's disease. Nature 1991;349:704-6.

5 Huntington's Disease Collaborative Research Group. A novel gene containing a trinucleotide repeat that is expanded and unstable on Huntington's disease chromosomes. Cell 1993;72:971-83.

6 Rosen DR, Siddique T, Patterson D, Figlewicz DA, Sapp P et al. Mutations in $\mathrm{Cu} / \mathrm{Zn}$ superoxide dismutase gene are associated with familial amyotrophic lateral sclerosis. Nature 1993;362:59-62.

7 Collinge J, Owen F, Poulter M, Leach M, Crow TJ, Rossor $\mathrm{MN}$, et al. Prion dementia without characteristic pathology. Lancet 1990;336:7-9.

8 Naber SP, Smith LL, Wolfe HJ. Role of the frozen tissue bank in molecular pathology. Diagn Mol Pathol 1992;1:73-9.

9 Everall IP, Luthert PJ, Lantos PL. Neuronal loss in the frontal cortex in HIV infection. Lancet 1989;i:309-11.

10 Cruz-Sanchez FF, Ravid R, Cuzner ML (eds). Neuropathological diagnostic criteria for brain banking. Oxford: IOS Press, 1995.

11 Mirra S, Heyman A, McKeel D, Sumi SM, Crain BS, Brownlee LM, et al. The consortium to establish a registry for Alzheimer's disease (CERAD). Part II. Standardization of the neuropathological assessment of Alzheimer's disease. Neurology 1991;41:479-86.

12 Chaudhuri KR, Crump S, al-Sarraj S, Anderson V, Cavan agh J, Leigh PN. The validation of the El Escorial criteria for the diagnosis of amyotrophic lateral sclerosis: a clinicopathological study. $\mathcal{F}$ Neurol Sci 1995;129(Suppl):11-12.

13 Litvan I, Hauw JJ, Bartko JJ, Lantos PL, Daniel SE, Horoupian DS, et al. Validity and reliability of the preliminar NINDS neuropathologic criteria for progressive supranuclear palsy. F Neuropathol Exp Neurol 1996;55:97-105.

14 Lantos PL. Neuropathological diagnostic criteria of multiple system atrophy. In: Cruz-Sanchez FF, Ravid R, Cuzner MI eds. Neuropathological diagnostic criteria for brain banking. Oxford: IOS Press, 1995:116-21.

15 Revesz T, Geddes JF, Daniel SE. Corticobasal degeneration. In: Cruz-Sanchez FF, Ravid R, Cuzner ML, eds. Neuropathological diagnostic criteria for brain banking. Oxford: IOS Press, 1995:99-104.

16 American Psychiatric Association Diagnostic and statistical manual of mental disorder, 4th edn. Washington, DC: American Psychiatric Association, 1994.

17 World Health Organization. International statistical classification of diseases and related health problems. 10th reviclassification of diseases and rela

18 College of American Pathologists. The systematized nomenclature of human and veterinary medicine (SNOMED). Northfield, Illinois: College of American Pathologists, 1993.

19 Dekaban AS, Sadowsky D. Changes in brain weights during the span of human life: relation of brain weights to body heights and body weights. Ann Neurol 1978;4:345-56.

20 Ravid R, Swaab DF. The Netherlands brain bank: a clinicopathological link in aging and dementia research. $f$ Neural pathological link in aging and de

21 Kingsbury A, Foster OJF, Nisbet AP, Cairns NJ, Bray L, Eve Kingsbury A, Foster OJF, Nisbet AP, Cairns NJ, Bray L, Eve
DJ, et al. Tissue $\mathrm{pH}$ as an indicator of mRNA preservation in human post-mortem brain. Mol Brain Res 1995;28:31118.

22 Health and Safety Commission. Control of substances hazardous to health regulations (COSHH). London: HMSO, 1994.

23 Clinical Pathology Accreditation (UK) Ltd. Accreditation handbook. Sheffield: Clinical Pathology Accreditation (UK) Ltd., 1995.

24 Health Services Advisory Committee. Safe working and the prevention of infection in the mortuary and post-mortem room. London: HMSO,

25 Bell JE, Ironside JW. How to tackle a possible Creutzfeldt Jakob disease necropsy. $\mathcal{f}$ Clin Pathol 1993;46:193-7.

26 Advisory Committee on Dangerous Pathogens. Precautions for work with human and animal transmissible spongiform encephalopathies. London: HMSO, 1994.

27 Advisory Committee on Dangerous Pathogens. Protection against blood-borne infections in the workplace. HIV and hepatitis. London: HMSO, 1995.

28 Advisory Committee on Dangerous Pathogens. Categorisation of biological agents according to hazard and categories of tion of biological agents according to hazard and categories of

29 Kozlowski PB. American brain banking network. $7 \mathrm{Neu}-$ ropathol Exp Neurol 1995;(Suppl):29S. 